\title{
Sonografiekurse nach den Richtlinien der ÖGUM
}

\section{detaillierte Infos im Kurs-Kalender unter: www.oegum.at/ausbildung/kurskalender.html}

\section{Abdomen}

- 21.-23.06.2017 Salzburg: Grundkurs Abdomen

Leitung \& Auskunft: OA Dr. Alois Hollerweger, kontakt@bhb-sonokurse.at

- 08.-09.09.2017 Burgenland: Abdomenultraschall Grundkurs nach OEGUM
Leitung \& Auskunft: OA Dr. Ybinger,

office@ultraschallkurse-wien.at

- 20.-22.09.2017 Salzburg: Aufbaukurs Abdomen

Leitung \& Auskunft: OA Dr. Alois Hollerweger, kontakt@bhb-sonokurse.at

- 28.-30.09.2017 Vorarlberg: Grundkurs Abdomensonografie
Leitung \& Auskunft: OA Dr. Otto Gehmacher, sekretariat.interne@vlkh.net

- 04.-06.10.2017 Linz: Grundkurs Abdomen

Leitung \& Auskunft: OA Dr. Schneider, kornelia.soellinger@ordensklinikum.at 
- 05.-07.10.2017 Wien: Grundkurs Abdomen

Leitung \& Auskunft: Univ. Prof. Dr. Gerhard Mostbeck, www.wienerultraschallseminare.at

- 19.-21.10.2017 Vorarlberg: Aufbauund Abschlusskurs Abdomensonografie

Leitung \& Auskunft: OA Dr. Otto Gehmacher, sekretariat.interne@vlkh.net

- 08.-09.11.2017 Baden: Ultraschallkurs Abdomen inkl. GI Trakt Leitung \& Auskunft: Univ. Prof. Dr. Gritzmann, norbert.gritzmann@gmail.com

- 15.-17.11.2017 Salzburg: Grundkurs Abdomen

Leitung \& Auskunft: OA Dr. Alois Hollerweger, kontakt@bhb-sonokurse.at

\section{Bewegungsapparat}

- 14.-16.09.2017 Innsbruck: MSUS Course for Rheumatologists - Basic Level

Leitung \& Auskunft: Priv.-Doz. Dr. Dejaco, margit.bonatti@chello.at

- 18.-20.10.2017 Graz: MUSCULOSKELETAL SONOGRAPHY COURSE IN RHEUMATOLOGY - INTERMEDIATE LEVEL

Leitung \& Auskunft: Priv.-Doz. Dr. Dejaco, noelle.tybery@klinikum-graz.at
Gefäße

- 10.-11.06.2017 Innsbruck: Transcranielle Doppler und Farbduplexsonografie Leitung \& Auskunft: Dr. Schmidauer, christine.span@tirol-kliniken.at

- 10.-11. 11.2017 Baden: Ultraschallkurs Halsgefäße und periphere Gefäße

Leitung \& Auskunft: Univ. Prof. Dr. Gritzmann, norbert.gritzmann@gmail.com

Notfallsonografie

- 29.06.2017 Baden: Ultraschallkurs für Intensiv und Notfallmediziner inkl. EFAST

Leitung \& Auskunft: Univ. Prof. Dr. Gritzmann, norbert.gritzmann@gmail.com

- 13.10.2017 Baden: Notfallsonografie EFAST Kurs

Leitung \& Auskunft: Univ. Prof. Dr. Gritzmann, norbert.gritzmann@gmail.com

Pädiatrie/ Bewegungsapparat

- 28.-29.06.2017 LKH Stolzalpe: Sonografie der Säuglingshüfte nach Graf Leitung \& Auskunft: Priv.-Doz. Dr. Tschauner, orthopaedie@lkh-stolzalpe.at

- 27.-28.09.2017 LKH Stolzalpe: Sonografie der Säuglingshüfte nach Graf Leitung \& Auskunft: Priv.-Doz. Dr. Tschauner, orthopaedie@lkh-stolzalpe.at
- 08.-09.11.2017 LKH Stolzalpe: Sonografie der Säuglingshüfte nach Graf Leitung \& Auskunft: Priv.-Doz. Dr.

Tschauner, orthopaedie@lkh-stolzalpe.at

Pädiatrie

- 15.-18.06.2017 Leoben: 26. Steirisches Seminar „Pädiatrische Ultraschalldiagnostik - Grundkurs“ Leitung \& Auskunft: OA Dr. Schweintzger, www.sonokolleg.at

- 13.-14.10.2017 Wien: Neonatale Schädelsonografie Leitung \& Auskunft: OÄ Dr. Zoder, office@neosono.at

- 09.-12.11.2017 Leoben: 26. Steirisches Seminar „Pädiatrische Ultraschalldiagnostik - Aufbaukurs“ Leitung \& Auskunft: OA Dr. Schweintzger, www.sonokolleg.at

Interdisziplinär

- 11.-13. Oktober 2017 Linz: 41. Ultraschalldreiländertreffen der ÖGUM/ DEGUM/SGUM „US 2017“

Weitere Informationen: www.ultraschall2017.at 